\title{
Contribution to the study of the chemical
} composition of Lemon Verbena: Aloysia triphylla (Hert). Britt. cultivated in Morocco

\section{Contribution à l'étude de la composition chimique de la Verveine odorante : Aloysia triphylla (L'Hert.) Britt cultivée au Maroc}

\author{
Abdeljalil Belkamel ${ }^{1}$, Jamal Bammi², Valérie Janneot ${ }^{3}$, Abdelfattah Belkamel $^{4}$, Youssef \\ Dehbi $^{4}$, Allal Douira ${ }^{2}$
}

\author{
${ }^{1}$ Faculté des Sciences et Techniques, Marrakech-Maroc. \\ ${ }^{2}$ Laboratoire de Botanique et de Protection des Plantes- Faculté des Sciences de Kénitra. Maroc. \\ ${ }^{3}$ Phytotagante, 9 boulevard de Clairfont, 66350 Toulouges France. \\ ${ }^{4}$ Nectarome, Tnine Ourika (Marrakech)-Maroc.
}

\begin{abstract}
By combining gas chromatography and mass spectrometry we have identified the main constituents of the essential oil of verbena tea (Alyosia triphylla) grown in Morocco. Harvests carried out at different stages of development of the plant allowed to characterize the qualitative variations of the active substances of the essential oil. The comparison of our results with the data of the literature gives some details on the different chemo types and the chemical diversity of this aromatic plant with high medicinal and economic value.
\end{abstract}

Keywords-Lemon Verbena, Morocco, verbena tea.

Résumé- En combinant la chromatographie en phase gazeuse et la spectrométrie de masse nous avons identifié les principaux constituants de l'huile essentielle de la verveine odorante (Alyosia triphylla) cultivée au Maroc. Les récoltes effectuées à différents stades de développement de la plante ont permis de caractériser les variations qualitatives des principes actifs de l'huile essentielle. La comparaison de nos résultats avec les données de la littérature apporte quelques précisions sur les différents chémotypes et la diversité chimique de cette plante aromatique à haute valeur médicinale et économique.

\section{INTRODUCTION}

La verveine odorante, Aloysia triphylla (l'hert.) Britt $=$ Lippia citriodora H.B. et K. appartient à la famille des Verbénacées. Elle est originaire du Chili et a été introduite en Europe vers 1784.

La verveine odorante est un arbuste vivace qui pousse rapidement et peut atteindre dans les meilleures conditions 2 à 3 mètres de hauteur. Ses tiges, droites ramifiées en branches étroites et minces, portent des feuilles entières et lancéolées, un peu rugueuses, caduques en hiver. Elles sont groupées par trois, d'où le qualificatif latin de triphylla. D'une couleur vert jaunâtre, elles dégagent un parfum citronné prononcé. Au cœur de l'été, de juillet à septembre, apparaissent à l'extrémité des tiges de longues grappes qui réunissent de nombreuses petites fleurs, blanches, violettes ou rougeâtres (Loaec, 2000).

La verveine est devenue une plante profondément ancrée dans la pharmacopée et l'art culinaire marocains. Elle est très cultivée, notamment, dans les régions de Marrakech, Agadir et Béni-Mellal, dans des jardins familiaux comme dans des plantations industrielles.

Ses cultures furent installées d'abord, vers le début des années 60, dans la région de Ghmat (environs de Marrakech), où elle prit la place du chanvre textile, puis dans la région d'Agadir. Elle occupe aujourd'hui plusieurs centaines d'hectares. Elle est surtout exportée 
séchée en vrac ou conditionnée en infusettes (Hadni, 1982).

Au cours des années 80 , sa culture occupe le premier rang dans l'exploitation agricole des régions d'Ourika, Ghmat et Mesfiwa (environs de Marrakech). $80 \%$ de la production en feuilles sèches est destinée à l'exportation. Les prix ont connu des augmentations considérables (100 $\mathrm{DH}$ le $\mathrm{Kg}$ de feuilles sèches). Sachant que les rendements sont de l'ordre de 1 à 2 tonnes à l'hectare. Les recettes peuvent être estimées de 100000 à $200000 \mathrm{DH}$ à l'hectare. En 2001-2002, le Maroc a exporté environ 437 tonnes de verveine séchée, ce qui représente une valeur d'environ 1.271.200 euros. Ce chiffre représente environ $4 \%$ de la quantité des plantes exportées et $9 \%$ de la valeur réalisée (Hadni, 1987).

Plusieurs auteurs ont rapporté les vertus thérapeutiques de la verveine odorante et de son huile essentielle. Selon Carnat et al. (1999), la verveine agit contre l'anxiété et l'insomnie. Elle est dotée aussi d'activités anti-oxydantes grâce à la présence dans sa composition de certains phénols (Zheng, 2001). Les feuilles ont un effet fébrifuge, antidouleurs nerveuses, antianémique et sont carminatives. La verveine est aussi tonicardiaque, hypoglycémiante et anti-migraine (Yousefzadeh et Meshkatalsadat, 2013).

Selon Roulier(1990), l'huile essentielle de verveine odorante est anti-inflammatoire articulaire et antirhumatismale. Elle est aussi calmante du système nerveux, antispasmodique, notamment dans les coliques hépatiques, antinévralgique et stimulant immunitaire en synergie avec d'autres huiles essentielles, tel que, le niaouli.

Sartoratto et al. (2004) ont mis en exergue le pouvoir antimicrobien de l'huile essentielle de la verveine odorante contre Enterococcus faecium, Salmonella cholerasuis et Candida albicans. Parodi et al. (2013) ont noté une inhibition modérée de l'HE de verveine odorante sur Aeromonas sp. Bien avant, Allegrini et al. (1973) et Belaich (1979) ont montré que l'HE de verveine présente une action inhibitrice sur Staphylococcus, Klebsiella, Neisseria et Escherichia coli.
Ali et al. (2011) ont signalé l'effet antibactérien intéressant de l'huile essentielle de verveine odorante contre Bacillus subtilis et Staphylococcus aureus et un effet antifongique partiel contre Phanerochaete chysosporium et Trichoderma reesei. Selon ces auteurs, ces effets antimicrobiens notables sont à corréler avec la richesse de cette huile essentielle en composés majoritaires dotés de pouvoir antimicrobien comme, entre autres, les citrals, le $\beta$-caryophyllène, le 1, 8-cinéole et le Citronellol.

Selon Belletti et al. (2004), l'effet inhibiteur des molécules aromatiques, comme le citral et le limonène, s'explique généralement par leur interaction avec les composants structuraux des cellules bactériennes. L'interaction avec la bicouche phospholipidique de la membrane cellulaire, entraîne une augmentation de la perméabilité et la fuite de constituants intracellulaires qui sont d'une importance vitale pour la bactérie (Singh et al., 2002).

Khani et al. (2012) ont mis en évidence l'effet inhibiteur de l'huile essentielle de la verveine odorante contre deux insectes: Tribolium confusum Jacquelin du Val et Callosobruchus maculatus (F.). Ces auteurs ont conclu que cette huile essentielle pourrait être utilisée comme agent de contrôle potentiel contre les insectes de produits stockés.

La présente étude s'insère dans une tendance multidisciplinaire qui tente à cerner les différents aspects culturaux et phytochimiques de la verveine odorante pour une meilleure optimisation de son utilisation dans les domaines pratiques, en l'occurrence dans le domaine thérapeutique et le domaine cosmétique.

\section{MATERIEL ET METHODE}

Afin de mieux connaître les caractéristiques de l'huile essentielle de la verveine odorante et afin de suivre les fluctuations de sa composition chimique, cinq échantillons d'huiles essentielles ont été distillés et analysés. 

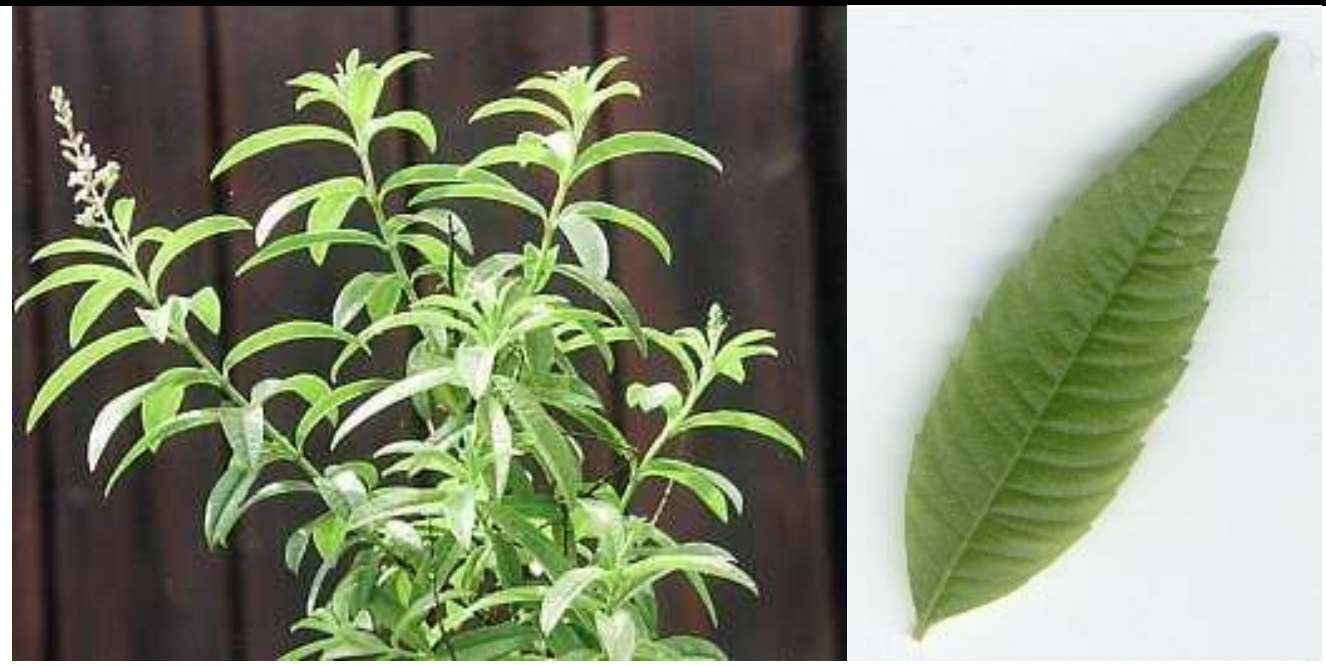

Fig.1: Verveine odorante en floraison

Ces échantillons sont issus de plantes provenant d'une parcelle qui se trouve à Tnine Ourika, à $35 \mathrm{~km}$ au sud-est de Marrakech (Maroc), à une altitude de $840 \mathrm{~m}$. La culture a été menée selon la méthode biologique sans utilisation de produits chimiques.

Une fois les coupes réalisées, la biomasse végétale est aussitôt transportée à la distillerie de la société Nectarome se trouvant à $400 \mathrm{~m}$ de distance du lieu de la culture. Les coupes ont eu lieu selon des dates précises (Tableau 1).

Tableau.1 : Calendrier des coupes de différents lots de verveine odorante

\begin{tabular}{|c|c|c|}
\hline $\begin{array}{c}\text { Numéro de } \\
\text { lots }\end{array}$ & $\begin{array}{c}\text { Date de } \\
\text { distillation }\end{array}$ & Origine \\
\hline 260603 & 26 Juin 2003 & Tnine ourika(Marrakech) \\
\hline 050703 & 05 Juillet 2003 & Tnine ourika(Marrakech) \\
\hline 120703 & 12 Juillet 2003 & Tnine ourika(Marrakech) \\
\hline 160703 & 16 Juillet 2003 & Tnine ourika(Marrakech) \\
\hline 200703 & 20 Juillet 2003 & Tnine ourika(Marrakech) \\
\hline
\end{tabular}

Les caractéristiques de distillation des différents lots de verveine sont les suivants :

- Partie distillée : sommités fleuries à l'état frais.

- Alambic en inox: capacité 1000 litres, équipé d'un essencier, lui aussi en inox, de capacité de 70 litres.

- Technique de distillation : entraînement à la vapeur d'eau.

- Source de la vapeur: chaudière délivrant 300 kilo vapeur à l'heure et fonctionnant avec du gasoil, l'eau est issue d'une station de pompage, puis passe par un adoucisseur avant d'accéder à la chaudière.

- Durée de la distillation : 2 heures.

- Rendement en huile essentielle : 0,15\% en moyenne. Les échantillons d'huiles essentielles obtenus ont été analysés en France au laboratoire de la société Phytotagante à Toulouges, en utilisant la chromatographie en phase gazeuse selon les conditions opératoires ci-dessous:

Chromatographe en phase gazeuse : HP5890

Détecteur : FID (Détecteur à ionisation de flamme)

Colonne : INNOWAX, $60 \mathrm{~m}, 32 \mathrm{~mm}, 0.5 \mathrm{~mm}$.

Isotherme initial : $60^{\circ} \mathrm{C}$.

Programmation : $2^{\circ} \mathrm{C} / \mathrm{min}$ jusqu'à $250^{\circ} \mathrm{C}(20 \mathrm{~min}$ au total).

Intégrateur : HP $3380 \mathrm{~A}$.

Volume injecté : $0.5 \mathrm{ml}$.

Gaz vecteur : Hélium ( $1 \mathrm{ml} / \mathrm{min}$ ).

\section{RESULTATS ET DISCUSSIONS}

La distillation des cinq échantillons a permis d'avoir des rendements comparables, qui tournent autour d'une moyenne de 0,15 \%. D'après Carnat et al. (1999), ce pourcentage varie entre 0,2 et $1 \%$.

L'étude des différentes huiles essentielles obtenues, a montré l'existence de quelques trente six composants appartenant à différentes familles chimiques. Le chromatogramme ci-après illustre la richesse quantitative de l'huile essentielle de verveine odorante analysée. 


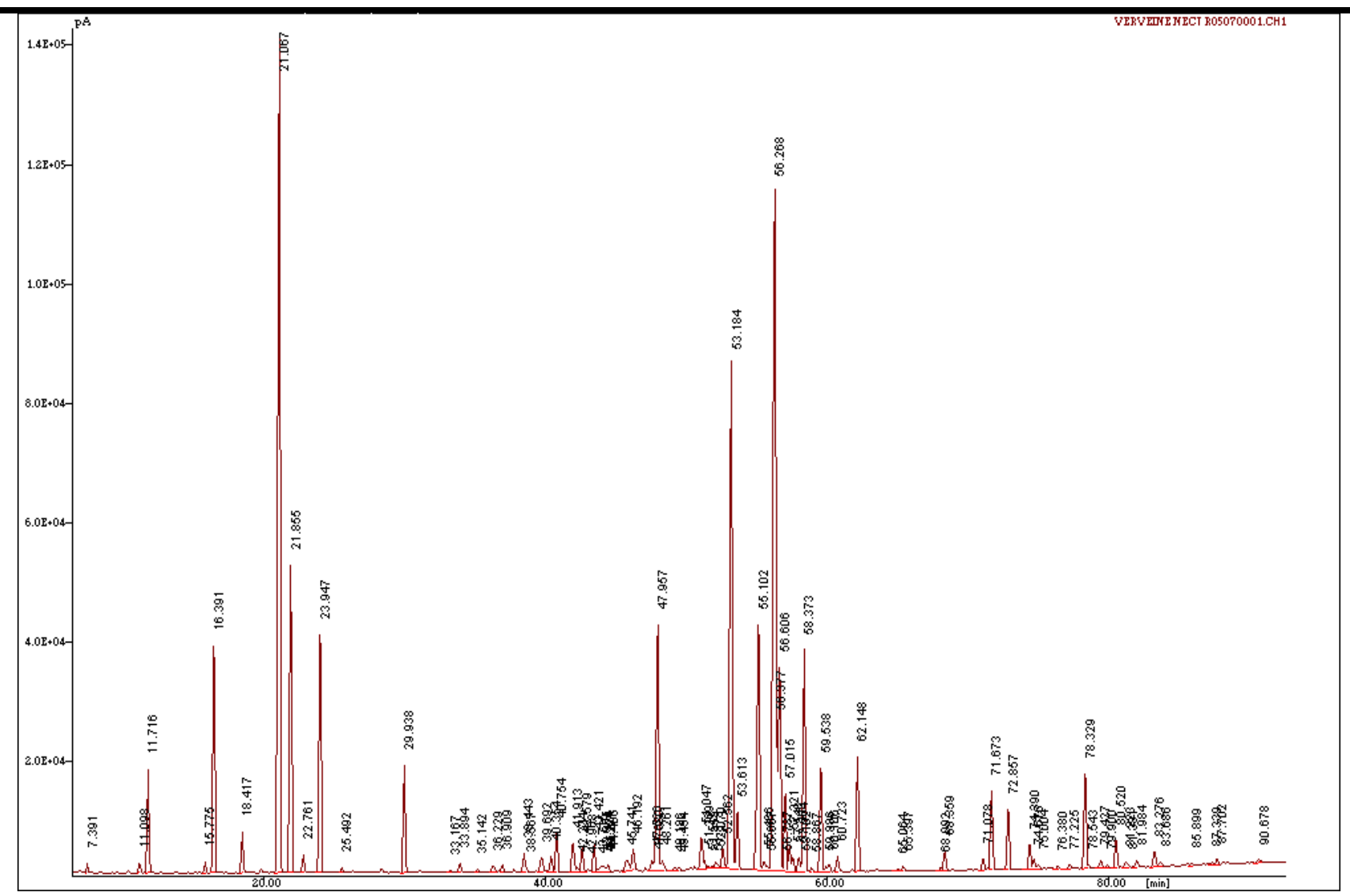

Fig.1: Chromatogramme de l'H.E de verveine, cas de l'échantillon 050703

Les composants identifiés de l'H.E de verveine odorante marocaine sont donnés dans le tableau 2:

Tableau.2: Liste des composants identifiés dans les cinq échantillons étudiés

\begin{tabular}{|c|c|c|c|c|c|}
\cline { 2 - 6 } \multicolumn{1}{c|}{} & \multicolumn{5}{c|}{ ECHANTILLONS D'H.E DE } \\
VERVEINE & $\mathbf{1 6 0 7 0 3}$ & $\mathbf{2 0 0 7 0 3}$ \\
\hline LOTS & $\mathbf{2 6 0 6 0 3}$ & $\mathbf{0 5 0 7 0 3}$ & $\mathbf{1 2 0 7 0 3}$ & $\mathbf{1 6 0 7}$ \\
\hline
\end{tabular}

\begin{tabular}{|c|c|c|c|c|c|c|}
\hline & Rendement en H.E & $0,12 \%$ & $0,15 \%$ & $0,2 \%$ & $0,1 \%$ & $0,17 \%$ \\
\hline Pics & composants & $\%$ & $\%$ & $\%$ & $\%$ & $\%$ \\
\hline 1 & Alpha- pinène + alpha- thujène & 1,33 & 1,19 & 1,16 & 1,24 & 1,19 \\
\hline 2 & Bêta- pinène & 0,15 & 0,13 & 0,13 & 0,13 & 0,13 \\
\hline 3 & sabinène & 3,08 & 2,67 & 2,49 & 2,8 & 2,73 \\
\hline 4 & myrcène & 0,54 & 0,46 & 0,43 & 0,39 & 0,49 \\
\hline 5 & limonène & 19,54 & 16,47 & 16,6 & 16,87 & 16,9 \\
\hline 6 & cinéole & 4,27 & 4,44 & 4,58 & 4,17 & 5,01 \\
\hline 7 & cis bêta- oïdium & 0,29 & 0,22 & 0,19 & 0,19 & 0,23 \\
\hline 8 & $\begin{array}{l}\text { trans. bêta- ocimène + gamma- } \\
\text { terpinène }\end{array}$ & 4,02 & 3,2 & 2,67 & 3,08 & 3,27 \\
\hline 9 & para cymène & 0,23 & 0,06 & 0,06 & 0,05 & 0,00 \\
\hline 10 & méthyl-6-hepten-5-one-2 & 1,84 & 1,44 & 1,39 & 1,64 & 1,72 \\
\hline 11 & rosefurane & 0,17 & 0,14 & 0,14 & 0,16 & 0,15 \\
\hline 12 & trans-4-thujanol & 0,24 & 0,29 & 0,26 & 0,24 & 0,36 \\
\hline 13 & citronnellol & 0,5 & 0,41 & 0,37 & 0,48 & 0,47 \\
\hline 14 & cis cis photocitral & 0,35 & 0,31 & 0,31 & 0,29 & 0,3 \\
\hline 15 & Alpha- copaène & 0,74 & 0,59 & 0,68 & 0,76 & 0,61 \\
\hline 16 & Bêta- bourbonène & 0,13 & 0,13 & 0,14 & 0,13 & 0,13 \\
\hline 17 & linalol & 0,4 & 0,39 & 0,37 & 0,39 & 0,38 \\
\hline
\end{tabular}




\begin{tabular}{|c|c|c|c|c|c|c|}
\hline 18 & terpinène-4-ol & 0,22 & 0,19 & 0,22 & 0,22 & 0,19 \\
\hline 19 & Bêta- caryophyllène & 6,05 & 4,81 & 5,19 & 5,4 & 4,72 \\
\hline 20 & Bêta- cédrène & 0,32 & 0,27 & 0,23 & 0,25 & 0,21 \\
\hline 21 & allo aromadendrène & 0,71 & 0,52 & 0,59 & 0,64 & 0,53 \\
\hline 22 & néral & 7,41 & 11,44 & 11,34 & 9,08 & $\mathbf{1 1 , 5 8}$ \\
\hline 23 & Alpha- terpinéol & 0,97 & 1 & 1,01 & 0,96 & 0,99 \\
\hline 24 & zingibérine + germacrène $\mathrm{D}$ & 5,95 & 5,01 & 4,64 & 4,86 & 5 \\
\hline 25 & géranial & 10,41 & 16,45 & 16,24 & 13,36 & 16,37 \\
\hline 26 & Acétate de géranyle & 4,75 & 3,99 & 3,17 & 4,1 & 3,68 \\
\hline 27 & $\begin{array}{l}\text { bicyclogermacrène + bêta- } \\
\text { curcumène }\end{array}$ & 1,29 & 1,19 & 1,06 & 1,21 & 1,05 \\
\hline 28 & cadinène & 0,32 & 0,22 & 0,3 & 0,28 & 0,22 \\
\hline 29 & ar. curcumène & 4,43 & 3,92 & 4,62 & 5,32 & 3,89 \\
\hline 30 & nérol & 1,66 & 1,71 & 1,52 & 1,5 & 1,55 \\
\hline 31 & géraniol & 1,77 & 1,9 & 1,77 & 1,83 & 1,8 \\
\hline 32 & oxyde d' isocaryophyllène & 0,21 & 0,2 & 0,24 & 0,25 & 0,19 \\
\hline 33 & oxyde de caryophyllène & 1,47 & 1,43 & 1,85 & 1,98 & 1,32 \\
\hline 34 & trans nérolidol & 0,96 & 0,88 & 0,86 & 1,1 & 0,86 \\
\hline 35 & spathulénol & 1,75 & 1,67 & 1,93 & 2,51 & 1,52 \\
\hline 36 & cadinol & 0,5 & 0,47 & 0,52 & 0,64 & 0,42 \\
\hline
\end{tabular}

Parmi les 36 composants identifiés, l'intérêt a été porté sur dix éléments ayant une concentration individuelle d'au moins 1,5 $\%$. L'ensemble des composants choisis représentent un pourcentage total moyen d'environ $68,5 \%$ de la masse totale de l'huile essentielle. L'étude portée sur un pourcentage en concentration de $68,5 \%$, est significative. Le tableau 3, donne la liste des éléments sélectionnés pour le reste de l'étude.

Tableau.3: Concentrations des principaux composants de cinq échantillons de l'huile essentielle de verveine odorante cultivée au Maroc

\begin{tabular}{|c|c|c|c|c|c|c|}
\hline Lots & 260603 & 050703 & 120703 & 160703 & 200703 & MOYENNE \\
\hline \multicolumn{7}{|l|}{ Composants } \\
\hline Limonène & 19,54 & 16,47 & 16,6 & 16,87 & 16,9 & 17,28 \\
\hline 1-8-cinéole & 4,27 & 4,44 & 4,58 & 4,17 & 5,01 & 4,49 \\
\hline$\beta$-caryopyllène & 6,05 & 4,81 & 5,19 & 5,4 & 4,72 & 5,23 \\
\hline Néral & 7,41 & 11,44 & 11,34 & 9,08 & 11,58 & 10,17 \\
\hline Zingibérine + germacrène $\mathrm{D}$ & 5,95 & 5,01 & 4,64 & 4,86 & 5,00 & 5,09 \\
\hline Géranial & 10,41 & 16,45 & 16,24 & 13,36 & 16,37 & 14,57 \\
\hline Acétate de géranyle & 4,75 & 3,99 & 3,17 & 4,1 & 3,68 & 3,94 \\
\hline Ar.curcumène & 4,43 & 3,92 & 4,62 & 5,32 & 3,89 & 4,44 \\
\hline Nérol & 1,66 & 1,71 & 1,52 & 1,5 & 1,55 & 1,59 \\
\hline Géraniol & 1,77 & 1,9 & 1,77 & 1,83 & 1,8 & 1,81 \\
\hline TOTAL & 66,24 & 70,14 & 69,67 & 66,49 & 70,5 & 68,61 \\
\hline
\end{tabular}

On constate que l'huile essentielle de verveine odorante se caractérise par une présence importante de citrals: le néral et le géranial qui représentent sur les 5 échantillons analysés une moyenne d'environ $24,75 \%$, avec $10,15 \%$ de néral et $14,6 \%$ de géranial. Leurs alcools correspondants, le nérol et le géraniol représentent de 3,5 $\%$ environ, avec $1,6 \%$ de nérol et $1,8 \%$ de géraniol.

Bellakhdar et al. (1994) ont analysé l'huile essentielle des sommités fleuries de la verveine odorante cultivée dans la région d'Agadir (Maroc) qui s'est avérée contenir 9,9\% de géranial, 6,9\% de néral, 7,4\% de 6-méthyl-5-hepten-2one et $12,4 \%$ de 1,8-cinéole. Ces résultats diffèrent des nôtres en ce qui concerne les citrals qui représentent $16,8 \%$ contre $24,75 \%$ dans la présente étude. Par contre, le 1-8 cinéole est mieux représenté dans l'échantillon d'Agadir (12, 4\%) que dans celui de Marrakech (Tnine Ourika) $(4,49 \%)$. 
Oukerrou et al. (2017) ont noté chez l'HE des feuilles de verveine de Ait Imour (région de Marrakech), le transcaryophyllène oxyde $(14,22 \%)$, le $\beta$-Spathulénol $(13,42 \%)$, l'Ar-curcumène $(11,30 \%)$ et le néral $(6,37 \%)$. A l'exception du néral qui reste, en général, comparable à nos résultats, les autres composants affichent des pourcentages trop éloignés des nôtres, c'est le cas notamment du $\beta$-Spathulénol avec $13,42 \%$ contre un pourcentage variant entre 1,52 et 2,51 dans notre étude. A signaler que la partie distillée par Oukerrou et al. (2017) est la feuille, tandis que dans notre étude, c'est les sommités fleuries qui ont été distillées. Ajoutons à cela, que Ait Imour se situe à une altitude de $1669 \mathrm{~m}$ contre seulement $840 \mathrm{~m}$ dans notre zone d'étude. Ces résultats mettent en évidence les variations biochimiques de l'huile essentielle en fonction de l'organe soumis à l'hydrodistillation et vraisemblablement sous l'effet de l'altitude. Cette approche comparative pourrait avoir un grand intérêt dans la caractérisation des différentes huiles essentielles de verveine provenant de différentes zones biogéographiques du Maroc et résultant de la distillation de différents organes de la plante.

Au Portugal, Santos-Gomes et al. (2005), ont mis en exergue des variations de la composition chimique de l'HE de verveine en fonction de l'organe distillé. Le géranial $(26,8-38,3 \%)$, le néral $(20,8-29,6 \%)$ et le limonène $(5,7-20,6 \%)$ sont présents dans les feuilles et dans les fleurs. Le 1-octène, le 1-octène-3-ol, le pcymène, le (Z)- $\beta$-ocimène et le trans-carvéol, ne sont identifiés que dans l'huile essentielle extraite des fleurs. Alors que le $\beta$-citronellène, le $\beta$-pinène, l'acétate de néryle et le trans-calaménène, ne se trouvent que dans l'huile essentielle issue des feuilles.

En Turquie, Özek et al. (1996) ont noté que l'HE de verveine issue des feuilles contient $14,8 \%$ de limonène et $17,9 \%$ de citrals alors que celle issue de branches feuillues contient $18,6 \%$ de limonène et $27,9 \%$ de citrals. Ibrahim et al. (2015) ont trouvé dans l'huile essentielle de verveine odorante cultivée en Egypte : le d-limonène (6.3 $-16,2 \%)$, le 1,8 cinéole $(4,7 \%-7,3 \%)$ et le citral $(19,9 \%$ $28,8 \%)$.
Hudaib et al. 2013, ont décelé dans l'HE de verveine cultivée en Jordanie du limonène $(17,7 \%)$, du géranial $(10,1 \%)$ et du néral $(9,8 \%)$. Ces derniers résultats sont proches des nôtres, notamment en ce qui concerne le limonène et les citrals.

Dans d'autres études, le taux de citrals atteint des niveaux élevés. C'est le cas notamment en Iran, avec un taux de géranial de 30,67 à 36,87\% et de néral entre 21,71 et 28,33\% (Shahhoseini et al. 2014).

En Argentine, Di Leo Lira et al. (2008), ont décelé une composition de l'HE de verveine, conforme au tableau chimique habituel: le néral $(20,0 \%)$ et le géranial $(29,0 \%)$. Dans d'autres échantillons analysés par ces auteurs, la présence de limonène et de citronellal à des teneurs élevés, respectivement de $40,3 \%$ et $21,6 \%$, dote cet échantillon d'une certaine originalité. Un autre échantillon contient une proportion inhabituelle de $\beta$ thujone qui atteint $73,4 \%$. Toujours en Argentine, Zygadlo et al. (1994) ont décelé une composition chimique originale à base de myrcénone $(36,50 \%)$, de l' $\alpha$ thujone $(13,10 \%)$, de la lippifoli-1 (6) -en-5-one $(8,87 \%)$ et du limonène $(6,87 \%)$.

Ces spécifications sont d'un grand intérêt pratique et pourraient être utilisées pour la standardisation de la verveine odorante, en caractérisant, entre autres, les variétés riches en substances toxiques, en l'occurrence le $\beta$-thujone.

A signaler aussi que le citronellol qui est très minoritaire dans notre étude, avec un moyen de $0,47 \%$, atteint, dans une autre étude, le pourcentage de $8.87 \%$ (Ali et al. 2012).

La figure 2, montre la présence de trois groupes de composés, dans les huiles essentielles analysées, se distinguant nettement sur le plan quantitatif. Le premier groupe est formé de limonène, néral, et géranial; le deuxième groupe est formé de cinéole-1-8, $\beta$ caryophyllène, zingibérine + germacrène $\mathrm{D}$, acétate de géranyle et Ar-curcumène; le troisième groupe est formé de nérol et géraniol. 


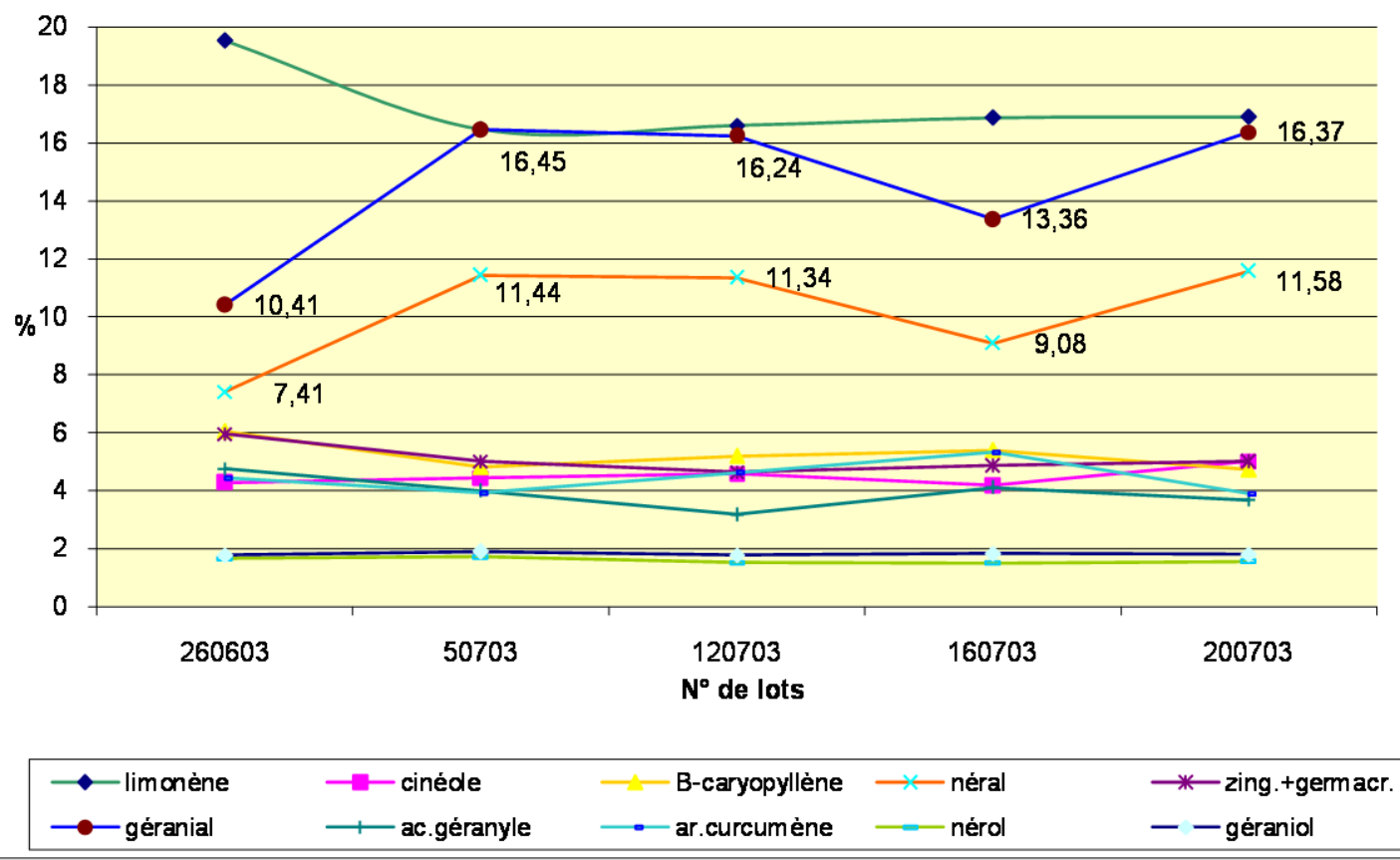

Fig.2: Evolution des concentrations de certains composants chimiques dans cinq échantillons d'H.E de verveine.

Pour le premier groupe, le limonène reste majoritaire par rapport aux autres composés étudiés et ce, pour l'ensemble des échantillons des H.E de verveine analysés. Le taux le plus élevé a été enregistré dans l'échantillon 260603 qui correspond à l'H.E distillée le 26 Juin 2003, il est de $19,5 \%$. Ce taux se stabilise après autour de $16 \%$ dans les autres échantillons des H.E analysées, numérotées comme suit: 050703, 120703, 160703 et 200703

Le géranial, qui appartient à la famille chimique des aldéhydes voit sa concentration fluctuer en fonction du temps et en fonction des échantillons analysés. En effet, sa concentration passe d'un minimum de 10,4\% dans l'échantillon 260603 à un maximum de 16,45\% dans l'échantillon 050703.

Le taux le plus faible est enregistré pendant le mois de juin, il se stabilise dans les deux échantillons des H.E du 05 et 07 Juillet 2003, avec une valeur autour de $16 \%$. La distillation réalisée 4 jours plus tard, c'est à dire le 12 Juillet 2003, montre une concentration du géranial dans l'H.E plus faible, elle est de l'ordre de $13 \%$. Ce taux retrouve la valeur de concentration qui est de $16 \%$ dans la dernière coupe réalisée le 20 Juillet 2003 et qui correspond au lot 200703. La moyenne générale de la concentration du géranial dans les 5 échantillons analysés est de $16,4 \%$ environ.

Quant au néral, appartenant lui aussi aux aldéhydes, il a subi des variations en fonction des périodes de coupes et de distillations. A noter que l'allure correspondant à l'évolution de sa concentration suit parfaitement celle du géranial tout au long de l'étude. Toutefois, cette concentration reste plus faible sur l'ensemble des échantillons étudiés. Sa moyenne sur les 5 échantillons est de $11,6 \%$ environ.

La concentration du néral est de 7,4\% environ au mois de Juin 2003, ce qui correspond à la concentration la plus faible enregistrée tout au long de l'étude. Cette concentration est rencontrée dans l'échantillon d'huile essentielle du lot 260603. Le pourcentage de ce composant évolue rapidement en une dizaine de jours pour atteindre environ $11,5 \%$ dans le lot 050703, correspondant à la distillation réalisée le 05 Juillet 2003. La concentration de ce composant se stabilise à environ $11,5 \%$ dans l'échantillon suivant qui correspond au lot 120703, il s'agit donc de la distillation qui a été effectuée une semaine plus tard.

L'analyse faite sur le lot d'H.E 160703, distillée 4 jours plus tard, montre une chute nette de la concentration de cet aldéhyde, elle atteint environ $9 \%$.

Dans le dernier lot analysé, référencé 200703, le taux de néral retrouve la valeur de 11,5\%.

Le deuxième groupe est formé des composants biochimiques suivants: le cinéole-1-8, le bétacaryophyllène, le zingibérine + germacrène $\mathrm{D}$, l'acétate de géranyle et l'ar-curcumène (Figure 2).

Ces cinq composés, montrent un comportement similaire vis à vis de leurs concentrations dans les échantillons d'H.E analysées. En effet, aucune variation significative en fonction du temps n'a été constatée. Les différentes concentrations oscillent entre $3 \%$ et $6 \%$ dans les 5 échantillons. Toutefois, on peut remarquer une légère relation de proportionnalité inverse entre le cinéole-1-8 et l'acétate de géranyle, comme le montre la figure 3 . On remarque, dans les 5 échantillons étudiés, que lorsque le 
cinéole-1-8 augmente, l'acétate de géranyle diminue et vis versa.

C'est dans le lot 120703 où on assiste à la différence la plus importante au niveau des concentrations de ces deux éléments, le cinéole-1-8 représente 4,58 \%, alors que l'acétate de géranyle représente $3,17 \%$, soit une différence de $1,41 \%$.
Il est à noter que malgré cette proportionnalité inverse, la concentration du cinéole 1-8 reste supérieure à celle de l'acétate de géranyle, sauf dans l'échantillon du 26 Juin 2003 où l'acétate de géranyle présente une concentration légèrement élevé de $4,75 \%$ contre $4,27 \%$ pour le 1-8cinéole. Ces résultats nous incitent à émettre l'hypothèse d'une relation biosynthétique éventuelle entre le cinéole et l'acétate de géranyle.

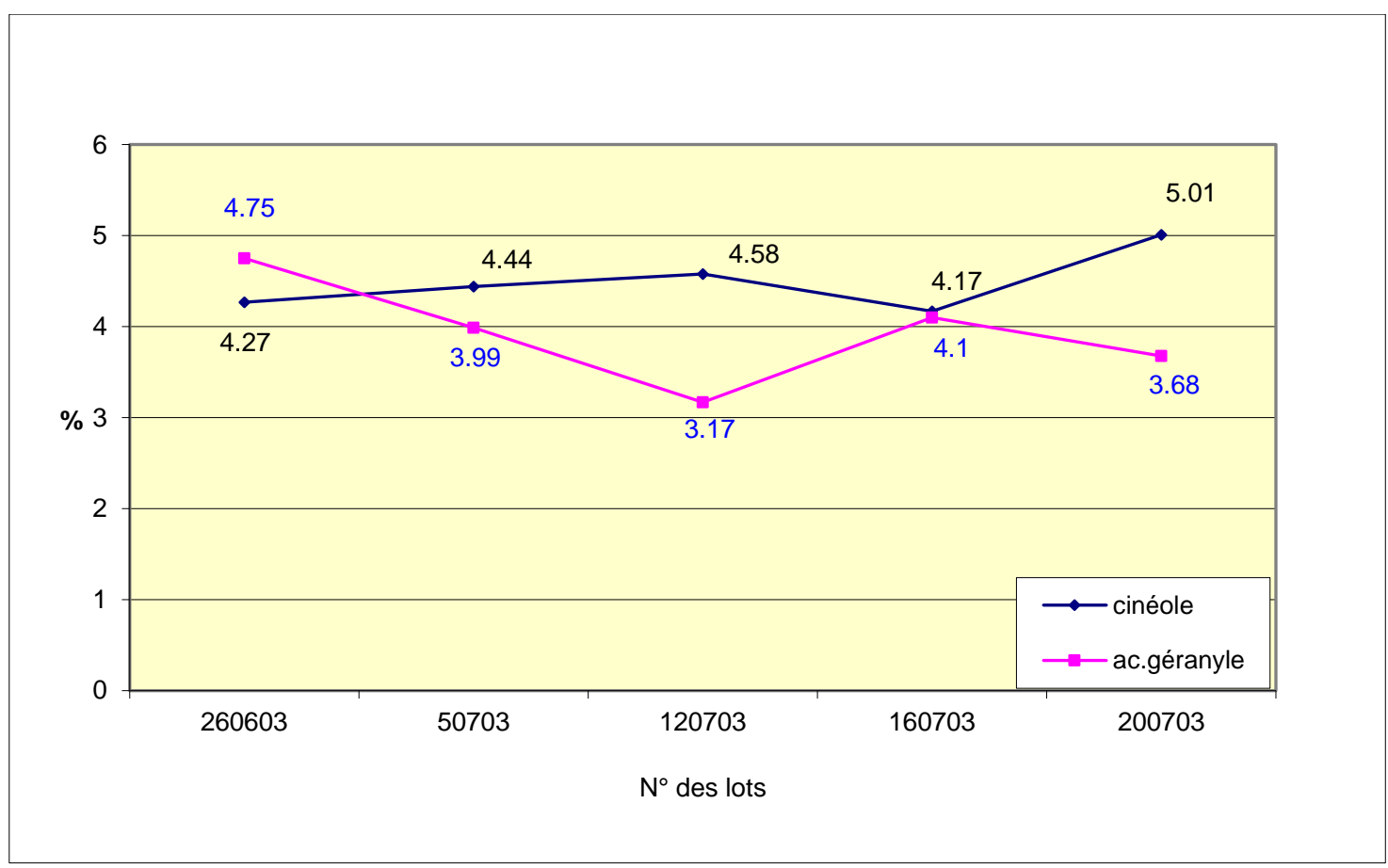

Fig.3: Comparaison et évolution des concentrations du 1-8-cinéole et de l'acétate de géranyle

Le troisième groupe est formé seulement de deux composés chimiques, à savoir le nérol et le géraniol, leur concentration reste stable autour de $1,7 \%$ en moyenne, dans les 5 échantillons analysés. Aucune variation n'a été constatée en fonction des coupes.

Dans la littérature, les variations chimiques qualitatives, en fonction de périodes de coupe, sont fréquentes. Dans cet ordre d'idées, Argyropoulou et al. (2007) ont décelé des différences entre l'HE des parties aériennes de verveine récoltées en mai, en pleine croissance, et en septembre, en pleine floraison. De mai à septembre, les taux de géranial et néral ont diminué respectivement de $38,7 \%$ à $26,8 \%$ et de $24,5 \%$ à $21,8 \%$ tandis que le taux de limonène a augmenté de $5,8 \%$ à $17,7 \%$.

Dans la même optique, Shahhoseini et al. (2013) ont analysé, en Iran, les parties aériennes de verveine récoltées à trois stades de croissance. Le géranial, enregistrant le taux le plus élevé au stade végétatif $(33,7 \%)$, a légèrement diminué en pleine la floraison $(32,7 \%)$. Le néral est passé de $26,1 \%$ au stade végétatif à $25,06 \%$ au stade fruitier.

D'autres facteurs, biotiques ou abiotiques, peuvent influencer la composition chimique de l'HE de la verveine odorante. Dans cet ordre d'idées, Schmidt et al. (2016) ont examiné l'effet du gel sur la composition de l'HE de verveine odorante cultivée au Brésil. Le limonène était le plus sensible au gel, représentant $14,36 \%$ des teneurs en huile avant et $10,15 \%$ après le gel.

Kassahun et al. (2011) ont montré que la biomasse foliaire et le rendement en HE de verveine, cultivée en Ethiopie, augmentent avec l'âge de la première à la deuxième année, au-delà de la troisième année ces paramètres commencent à décliner. Prochnov et al. (2017) ont noté que les citrals enregistrent les taux les plus faibles en hiver et les taux les plus élevés en automne et en été.

Les conditions culturales peuvent aussi influencer la composition chimique de l'huile essentielle de verveine odorante. Dans cette optique, Moein et al. (2014) ont montré que la culture sous serre favorise la synthèse des citrals. Agah et Najafian (2013) ont montré que le séchage à l'ombre augmente le rendement en $\mathrm{HE}$ et favorise la synthèse de limonène, néral et géranial.

L'analyse des huiles essentielles de la verveine odorante, a révélé l'existence de deux groupes particuliers de composants chimiques, ayant des comportements 
inverses, c'est à dire, quand la concentration du premier s'élève, celle du second diminue et vis versa (tableau 4 et

Figure 4).

Tableau.4 : Les des deux groupes de composés à comportements inverses

\begin{tabular}{|l|c|c|c|c|c|}
\cline { 2 - 7 } \multicolumn{1}{c|}{ Lot analysé } & Lot 26-06-03 & Lot 05-07-03 & Lot12-07-03 & Lot16-07-03 & Lot20-07-03 \\
\hline (groupe A) \% & 40,79 & 47,97 & 47,97 & 42,64 & 48,2 \\
\hline (groupe)\% & 25,45 & 22,17 & 22,2 & 23,85 & 22,3 \\
\hline
\end{tabular}

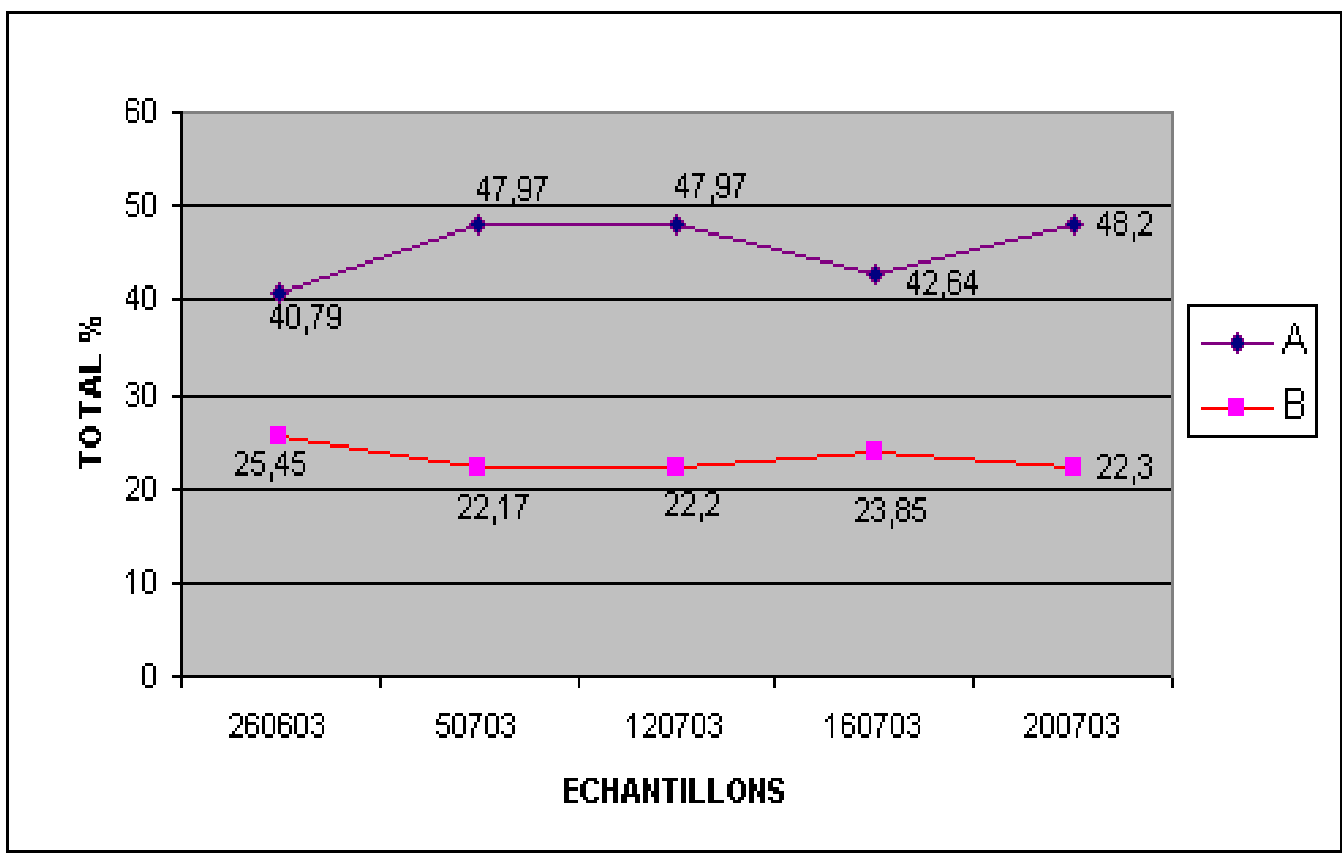

Fig.4: Evolution des concentrations de deux groupes de composés chimiques dans l'H.E de verveine odorante

La figure 4 montre que la courbe A, représente l'ensemble des composés suivants : limonène, néral, géranial, nérol et géraniol, alors que la courbe $\mathrm{B}$, représente l'ensemble des composés suivants: 1-8-cinéole, béta-caryophyllène, zingibérine + germacrène $\mathrm{D}$, acétate de géranyle, et arcurcumène.

Toutefois, ces fluctuations sont beaucoup plus accentuées dans la courbe A par rapport à la courbe B. Par ailleurs, on note la présence de 3 points de concentrations maximales dans la courbe $\mathrm{A}$, qui correspondent aux coupes du 05, du 12 et du 20 juillet, ces concentrations tournent autour de $48 \%$ environ, alors que pour la courbe $\mathrm{B}$, ces mêmes points correspondent plutôt à des concentrations minimales, qui tournent autour de $22 \%$ et inversement, les deux points de concentrations minimales relevées par la courbe $\mathrm{A}$ et qui tournent autour d'une moyenne de $41,7 \%$, correspondent aux deux points de concentrations maximales repérées dans la courbe B et dont la moyenne est d'environ 24,6\%.

Cette inversion au niveau des différentes concentrations étudiées, montrent un vraisemblable phénomène biosynthétique entre les deux groupes de composés chimiques (A et B).
Dans la littérature des phénomènes similaires ont été mis en évidence. En effet, Gomez et al. (2006), ont montré que les niveaux de géranial qui ont évolué d'environ $40 \%$ en septembre jusqu'à $27 \%$ en novembre, ont connu une augmentation par la suite, en corrélation inverse avec les taux d'oxyde de caryophyllène et d'ar-curcumène.

Toutefois, il reste à élucider ce «phénomène » de la relation biosynthétique qui existe entre les différentes molécules de cette huile essentielle.

\section{CONCLUSION}

D'après ce qui précède, on peut soupçonner l'existence d'une certaine relation biosynthétique entre les différents composants chimiques de l'huile essentielle de verveine analysée dans notre étude. L'ensemble des composants du groupe $\mathrm{A}$, présente une proportionnalité inverse avec le groupe des composants du groupe B, ceci montre bien une certaine relation entre les composants et une possibilité de transformation chimiques des molécules tout au long des saisons. De même, on a bien noté le caractère inverse des concentrations entre le 1-8-cinéole et l'acétate de géranyle dans les 5 échantillons analysés.

Etant donné que les aldéhydes ont des propriétés bactéricides, il devient intéressant, principalement dans le 
domaine de l'aromathérapie, de faire la coupe de la verveine odorante de la région d'étude et de pratiquer sa distillation à partir de la fin de la première semaine de juillet, ceci procure à l'huile essentielle une forte concentration en aldéhydes, en l'occurrence le groupe des citrals, composé du néral et du géranial.

Nasser Al-Deen et al. (2015) en analysant les huiles essentielles de verveine odorante cultivée en Syrie, ont montré que les citrals augmentent en juillet, ce qui dote l'HE extraire des feuilles récoltées pendant ce mois d'un pouvoir antibactérien plus important contre Escherichia Coli. C'est, d'ailleurs, à la richesse en citrals (géranial et néral) qu'est rapportée l'activité bactéricide (El Aziz, 1991).

D'autres études ont mis en exergue que le néral et le géranial pourraient induire l'apoptose dans la leucémie lymphoïde chronique (De Martino et al., 2009).

A noter aussi que le taux, relativement élevé, de zingibérine et germacrène $\mathrm{D}$ (environ $5 \%$ ) dote la verveine odorante de notre zone d'étude d'une certaine originalité et incite à pousser la recherche dans ce sens.

Il est donc fondamentale de bien connaître la meilleure période de coupe et de distillation, afin de permettre une utilisation optimale de cette huile essentielle dans les différents domaines d'applications.

Les divergences quantitatives et qualitatives enregistrées dans l'huile essentielle de verveine odorante cultivée de part le monde incitent, sur le plan pratique et appliqué, à bien caractériser les chémotypes et les conditions culturales pour bien potentialiser les utilisations de cette plante aromatique dans les domaines thérapeutiques et cosmétiques.

\section{RÉFÉRENCES}

Agah M. \& Najafian S. (2013). Essential oil content and composition of Lippa citriodora as affected by drying method before flowering stages. Journal of Medicinal Plants and By-products, 2: 205-208.

Ali H.F.M., El-Beltagi H.S. \& Nasr N.F. (2011). Evaluation of antioxidant and antimicrobial activity of Aloysia triphylla. EJEAF Che, 10(8) : 2689-2699.

Allegrini J., Simeon M. et Laillots H. (1973). Emulsion des huiles essentielles, fabrication et application en microbiologie. Travaux de la société de pharmacie de Montpellier, 33(1): 73-86.

Argyropoulou C., Dimitra Daferera D., Petros A.Tarantilis P.A, Costas Fasseas C., \& Polissiou M. (2007). Chemical composition of the essential oil from leaves of Lippia citriodora H.B.K. (Verbenaceae) at two developmental stages. Biochemical Systematics and Ecology, 35: 831-837.

Belaich P. (1979). Traité de phytothérapie et d'aromathérapie. Tome 1. Aromatogramme.
Bellakhdar J., Il Idrissi A., Canigueral S., Iglesias J. \& Vila R., (1994). Composition of Lemon Verbena (Aloysia triphylla (L'Herit.) Britton) oil of Moroccan origin. Journal of Essential Oil Research, 6: 523-526.

Bellakhdar. J. (1997). Pharmacopée marocaine traditionnelle. IBIS PRESS, Paris, France

Belletti N., Ndagijimana M., Sisto C., Guerzoni M.E., Lanciotti R. \& Gardini F. (2004). Evaluation of the antimicrobial activity of citrus essences on Saccharomyces cerevisiae. J. Agric. Food Chem., 52: 6932-6938.

Carnat A., Carnat A.P., Fraisse D \& Lamaison J.L. (1999). The aromatic and polyphenolic composition of lemon verbena tea. Fitoterapia, 70: 44-49.

De Martino L., D'Arena G., Minervini M.M \& al. (2009). "Verbena officinalis essential oil and its component citral as apoptotic inducing agent in chronic lymphocytic leukemia," International Journal of Immunopathology and Pharmacology, 22(4): 1097-1104.

Di Leo Lira P., Van Baren C.M, Retta D., Bandoni A.L, Gil A., Gattuso M. \& show all. (2008). Characterization of Lemon Verbena (Aloysia citriodora Palau) from Argentina by the Essential Oil. Journal of Essential Oil Research (20): 350-353.

El Aziz M. (1991). Activité antibactérienne des huiles essentielles des plantes aromatiques à citronellalcitronellol et/ou citral. D.E.S., Fac. des Sciences, Université Mohamed V, Rabat, maroc, 125p.

Gomes P.C.S., Oliveira, H.R.C., Vicente, A.M.S. \& Ferreira, M.F. (2006). Production, transformation and essential oils composition of leaves and stems of lemon verbena (Aloysia triphylla (L'Herit.) Britton) grown in Portugal. Rev. Bras. Pl. Med., Botucatu, 8: 130-135.

Hadni D. (1982). Contribution à l'étude technique et socioéconomique de la verveine. Mémoire de second cycle. IAV Hassan-II. Agadir-Rabat.

Hadni, D. (1987). Importance économique et aspect agronomique de la culture de la verveine dans la région de Marrakech: document du séminaire sur le dépérissement de la verveine dans la région de Marrakech, ORMVAH., Maroc.

Hudaib M., Tawaha K. \& Bustanji Y. (2013).Chemical Profile of the Volatile Oil of Lemon verbena (Aloysia citriodora) Growing in Jordan. Journal of Essential Oil Research.,16: 568-574.

Ibrahim M. E., Mohamed M. A. \& Khalid K A.,(2015). Growth and essential oil composition affected by foliar nutrition application on lemon verbena plant. J. Mater. Environ. Sci. 6 (7) pp: 1824-1828.

Kassahun B.M., Yosef W.B \& Mekonnen S.A. (2013). Performance of Lemon Verbena (Aloysia triphylla L.) for morphological, economic and chemical traits in Ethiopia. American-Eurasian J. Agric. \& Environ. Sci., 13 (11): 1576-1581. 
Khani A., Basavand F. \& Rakhshani E. (2012). Chemical composition and insecticide activity of lemon verbena essential oil. J. Crop Prot. 1 (4): pp313-320.

Loaec. M..H. (2000). Aromatiques - Quand cultiver son jardin. Ed. Hachette: pp : 116-117

Moein M., Mohammad M., Zarshenas M.M and Etemadfard H. (2014). Essential oil composition and total flavonoid content of Aloysia citriodora under different cultivation systems. International Journal of Plant, Animal and Environmental Science, (4): 353-358.

Naser AL-Deen M.G, Mansoor R. \& AL-Joubbeh M. (2015). Fluctuations of chemical composition of essential oil and Antimicrobial of Lemon verbena (Lippia Citriodora) during growth stages in Syria. International Journal of Chem Tech Research., 8(6): 704-710.

Oukerrou M.A, Tilaoui M., Hassan Ait Mouse H., Leouifoudi I., Jaafari A., \& Zyad A. (2017). Chemical Composition and Cytotoxic and Antibacterial Activities of the Essential Oil of Aloysia citriodora Palau Grown in Morocco. Advances in Pharmacological Sciences, Article ID 7801924, 10 pages.

Özek N. T. Kirimer K. H.C. Baser \& Tümen G. (1996). Composition of the Essential Oil of Aloysia triphylla(L'Herit.) Britton Grown in Turkey. Journal of Essential Oil Research, 8: 581-583

Parodi T.V., Vargas A.P. D. C., Krewer C., Flores E. M. D. M. \& al., (2013). Chemical composition and antibacterial activity of Aloysia triphylla (L'Hérit) Britton extracts obtained by pressurized $\mathrm{CO} 2$ extraction. Brazilian archives of biology and technology, 56(2): 283292.

Prochnow D., AltissimoB.S., Da Silva J.C., Meira D., Braulio Otomar Caron B.O., Berta Maria HeinzmannB.M. \& Denise Schmidt D. (2017). Chemical composition of the essential oil of Aloysia triphylla (L'Hér) Britton due to water deficit and seasonality. Boletín Latinoamericano y del Caribe de Plantas Medicinales y Aromáticas 16 (2): pp121-128.

Roulier. G. (1990). Les huiles essentielles pour votre santé. Ed. Dangles, pp: 306-308.

Santos-Gomes P.C, Fernandes-Ferreira M. \& Vicente A.M.S. (2005). Composition of the essential oils from flowers and leaves of Vervain [Aloysia triphylla (L'Herit.) Britton] Grown in Portugal. Journal of Essential Oil Research., 17: 73-78.

Sartoratto A., Machado A.L.M., Delarmelina C., Figueira G.M., Duarte M.C.T. \& Rehder V.L.G. (2004). Composition and antimicrobial activity of essential oils from aromatic plants used in Brazil. Brazilian Journal of Microbiology, (35): 275-280.

Schmidt D., Caron B.O., Prochnow D., Cocco C., Elli E.F., Stolzle J., Altissimo B. and Heinzmann B.M. (2016). Effect of frost on yield and composition of
Aloysia triphylla essential oil. Journal of Medicinal Plants Research, 10(7): 88-92.

Shahhoseini R., Arman Beyraghdar A., Karimi S-R \& Taghi Ebadi M. (2013). Essential Oil Content and Composition of Lemon Verbena (Lippia citriodora Kunth.) during Different Phenological Stages. Journal of Medicinal Plants and By-products, 2: 205-208.

Shahhoseini R., Hosseini N. \& Ghorbanpour M. (2014). Study of Essential Oil Content and Composition of Different Parts of Lemon verbena (Lippia citriodora) Grown in Iran. Journal of Essential Oil Bearing Plants, 17: $120-125$.

Singh N., Singh R.K., Bhunia A.K. \& Stroshine R.L. (2002). Efficacy of chlorine dioxide, ozone and thyme essential oil or a sequential washing in killing $E$. coli O157:H7 on lettuce and baby carrots. Lebensm.Wissu.Technol., 35: 720-729.

Yousefzadeh N. \& meshkatalsadat M.H. (2013). Quantitative and qualitative study of bioactive compounds of essential oils of plant Lippia citriodora by use of GCMS technique. Journal of Novel Applied Sciences, 2(2): 964-968.

Zheng W \& Wang S.Y. (2001). Antioxidant activity and phenolic compounds in selected herbs. J. Agric. Food Chem.,49: 5165-5170.

Zygadlo J.A, Lamarque A.L., Maestri D.M., Guzmán C.A., Lucini E.I., Grosso N.R. \& al. (1994). Volatile Constituents of Aloysia triphylla (L'Herit.) Britton. Journal of Essential Oil Research, 6: 407-409. 